\title{
X-ray variability in LINERs
}

\section{Josefa Masegosa ${ }^{1}$, Lorena Hernández-García ${ }^{1}$, Isabel Márquez ${ }^{1}$ and Omaira González-Martín ${ }^{2}$}

\author{
${ }^{1}$ Instituto de Astrofísica de Andalucía, CSIC, Granada, Spain \\ email: pepa@iaa.es \\ ${ }^{2}$ Instituto de Astrofísica de Canarias, La Laguna, Tenerife, Spain
}

\begin{abstract}
One of the most important features in active galactic nuclei (AGN) is the variability of their emission. Variability has been discovered at X-ray, UV, and radio frequencies on time scales from hours to years. Among the AGN family and according to theoretical studies, LowIonization Nuclear Emission Line Region (LINER) nuclei would be variable objects on long time scales. Our purpose is to investigate spectral X-ray variability in LINERs and to understand the nature of these kinds of objects, as well as their accretion mechanism. Chandra and XMMNewton public archives were used to compile X-ray spectra of LINER nuclei at different epochs with time scales of years. To search for variability we fit all the spectra from the same object with a set of models, in order to identify the parameters responsible for the variability pattern. We found that long term spectral variability is very common, with variations mostly related to hard energies (2-10 keV). These variations are due to changes in the soft excess, and/or changes in the absorber, and/or intrinsic variations of the source.
\end{abstract}

Keywords. Galaxies: active - X-rays: galaxies

\section{Introduction}

X-ray data for Low Luminosity Active Galactic Nuclei (LLAGN) offer the most reliable probe of the high-energy spectrum, providing many AGN signatures (D'Onofrio et al. 2012). In Low Ionisation Nuclear Emission Line Regions (LINERs) it can be assessed that an AGN is present when a point-like source is detected at hard X-ray energies (Satyapal et al. 2004, 2005; Dudik et al. 2005; Ho 2008). The most extensive work in this subject has been carried out by González-Martín et al. (2009), who analyzed 82 LINERs with Chandra and/or XMM-Newton data for single period observations. They found that $60 \%$ of the sample showed a compact nuclear source in the $4.5-8 \mathrm{keV}$ band; a multiwavelenght analysis resulted in about $80 \%$ of the sample showing evidence of AGN-related properties, which is a lower limit since Compton-thick $\dagger$ objects were not taken into account.

Variability is one of the main properties that characterizes AGN, most of these being at least mildly variable. At X-ray frequencies many studies have been made to understand variability in Seyfert galaxies (Risalitti et al. 2010, Evans et al. 2005, González-Martín \& Vaughan 2012, among others) and it has been concluded that they are indeed variable sources, althought the reason for X-ray variability in Seyferts of different types are still a matter of current studies. The first clear evidence of variability in LINERs was reported by Maoz et al. (2005) at UV frequencies, where all but three objects in their sample of 17 type 1 and 2 LINERs appeared variable. The works by Pian et al. (2010), Younes et al. (2011), González-Martín et al. (2011) and Hernández-García et al. (2013) at Xray energies agree with the consideration of variability being a common property of LINERs. It is important, however, to fully characterize both the scale and magnitude of

$\dagger$ Objects whose nucleus is obscured by large amounts of gas and dust, with $N_{H}>10^{24} \mathrm{~cm}^{-2}$. 
the variability. The work we present here shows the preliminary results on a systematic analysis of X-ray variability in LINERs.

\section{Sample and data}

We used the sample from the Palomar Spectroscopic Survey of neaby galaxies (Ho et al. 1997), which is the largest sample of this kind of galaxies with optical spectra, containing Seyferts, LINERs, and transition objects. The objects clasified as L1, L2 and L/S were selected. All these targets were searched for in the publicly archives of $X M M$-Newton and Chandra up to October 2013. 89 galaxies were found to have data on them (22 of type 1 and 67 of type 2). We then excluded objects affected by a pileup fraction larger than $10 \%$ and observations with less than 400 number counts to guarantee a proper spectral fitting. Finally LINERs with useful observations at more than one epoch were selected to study variability. For this work we study a sample of LINERs containing 16 objects, nine of type 2 and seven of type 1 (types 1.8 or 1.9 in Ho et al. (1997)) LINERs.

\section{Data analysis}

To obtain information on the parameters responsible of the observed variability, a modeling of the X-ray spectral energy distribution was performed as described in GonzálezMartín et al. (2009). The most general used baseline model that better fit LINER spectra is a composite of a thermal plus a power-law model, where the free parameters are the column densities, $N_{H 1}$ and $N_{H 2}$, the temperature, $k T$, the slope of the power-law, $\Gamma$, and the normalizations, Norm $_{1}$ and Norm $_{2}$ (MEPL in Table 1). As it can be seen in Table 1 for some particular cases an additional scattered power-law component may be needed to properly fit the soft energy bands, coded as $2 \mathrm{PL}$. To analize the X-ray variability of the sources, we have followed the methodology explained in Hernández-García et al. (2013). The spectral fitting process was done by using XSPEC version 12.7.0 in two steps: (1) individual analysis of each observation to determine the best fit for each spectrum and selection of the best baseline model (SMF0 in Table 1), and (2) simultaneous fitting of the set of spectra of the same object at different epochs.

For non variable sources SMF0 produces a proper fit for all the spectra. If this is not the case we let vary individually the parameters $N_{H 1}, N_{H 2}, \Gamma, \mathrm{Norm}_{1}, \mathrm{Norm}_{2}$, and kT (hereinafter SMF1 in the tables). Among these, we chose the best fit as that with $\chi_{r}^{2}=$ $\chi^{2} /$ d.o.f closest to unity and that improved the SMF0 fit (by using the F-test). The result of SMF1 was used as the baseline model for the next step. Finally, we also included the possibility that two parameters could vary together to explain the variability of the sources. For that purpose we fitted each set of data, letting the parameter found as the best fit in SMF1 vary together with any of the other parameters of the fit (hereinafter SMF2). Again the $\chi_{r}^{2}$ and F-test were used to determine whether this further complexity of the spectral fitting results in a significant improvement of the final fit. In Table 1, columns 8 and 9 , we include the parameter varying. When empty, it means that the simultaneous fitting with the SMF0 baseline model produces a good representation of all the observations, and thus no variations have been detected.

\section{Results and conclusions}

A simultaneous spectral analysis was performed for all our 16 sample LINERs.

Together with the nine LINERs 2 in our sample, we also consider two additional objects from Hernández-García et al. (2013) (see Table 1). Among these 11 LINERs 2, five 
show indeed spectral variability linked mostly to spectral variations at hard energies. Only NGC 4552 does vary at soft energies as well. The reason for such variability is due to variations in $\mathrm{Norm}_{1}$. Column 2 in Table 1 shows objects classified as AGN candidates (González-Martín et al. 2009). The three objects classified as non-AGN candidates (NGC 3608, NGC 5813, and NGC 5846) are compatible with no spectral variations, as expected. Considering AGN candidates (eight LINERs 2), five are variable.

For the seven LINERs 1 analyzed, we found variability in five of them, in all the cases linked to hard energies. We only found one galaxy where variations can be atributed to the spectral power-law slope, NGC 4636 (the only one classified as non-AGN, whose Chandra image contains a number of point-like sources within the $X M M$-Newton aperture). Among the six AGN candidates, four of them are variable, in two cases variations are needed in $\mathrm{Norm}_{2}$ and $N_{H 2}$, and in the other two only Norm 2 variations need to be invoked.

All together, X-ray variability has been found in $64 \%$ of the analyzed AGN candidates LINERs (9 out of 14), being equally frequent in type 1 LINERs (4 out of 6 ) and in type 2 LINERs (5 out of 8). No differences are found in the spectral variability, being in both cases linked to hard energies. We did found $N_{H 2}$ variability only in two out of the nine variable sources, none of them being a Compton-thick candidate. There does not seem to exist any clear relation with either the black hole mass or the Eddington ratios (see Columns 5 and 6 in Table 1). Thus we may conclude that variability is more related to the energy power of the AGN irrespectively of the LINER type nature.

\begin{tabular}{|c|c|c|c|c|c|c|c|c|c|}
\hline $\begin{array}{l}\text { Name } \\
(1)\end{array}$ & $\begin{array}{c}\text { Type } \\
(2)\end{array}$ & $\begin{array}{c}\log L_{\text {soft }} \\
(0.5-2 \mathrm{keV}) \\
(3)\end{array}$ & $\begin{array}{c}\log L_{\text {hard }} \\
(2-10 \mathrm{keV}) \\
(4)\end{array}$ & $\begin{array}{c}\log M_{B H} \\
\left(M_{\odot}\right) \\
(5)\end{array}$ & $\begin{array}{c}\log \left(R_{E d d}\right) \\
(6)\end{array}$ & $\begin{array}{c}\mathrm{SMF} 0 \\
(7)\end{array}$ & $\begin{array}{c}\text { Variability } \\
\text { SMF } 1 \\
(8)\end{array}$ & $\begin{array}{c}\text { SMF } 2 \\
(9)\end{array}$ & $\begin{array}{c}\mathrm{T} \\
(\text { Years) } \\
(10)\end{array}$ \\
\hline \multicolumn{10}{|c|}{ Type 2} \\
\hline NGC $1961(\mathrm{X})$ & & 41.20 & 41.23 & 8.67 & -4.03 & ME2PL & - & - & 0.08 \\
\hline NGC $3608^{*}(\mathrm{X})$ & Non-AGN & 40.32 & 40.24 & 8.06 & -4.41 & ME2PL & - & - & 12 \\
\hline NGC $4261(\mathrm{X})$ & $\mathrm{AGN}$ & 40.98 & 41.02 & 8.96 & -4.54 & ME2PL & - & - & 8 \\
\hline$(\mathrm{C})$ & & & & & & ME2PL & - & - & 6 \\
\hline NGC $4374^{*}(\mathrm{C})$ & $\mathrm{AGN}$ & 39.64 & 39.59 & 8.74 & -5.79 & MEPL & $\mathrm{Norm}_{2}$ & - & 5 \\
\hline NGC $4494(\mathrm{X}, \mathrm{C})$ & $\mathrm{AGN}$ & 39.13 & 40.75 & 7.64 & -2.38 & PL & Norm & - & 0.3 \\
\hline NGC $4736(\mathrm{X})$ & $\mathrm{AGN}$ & 39.61 & 39.73 & 6.98 & -3.84 & MEPL & - & - & 4 \\
\hline NGC 5195 (X) & $\mathrm{AGN}$ & 39.24 & 39.25 & 7.59 & -4.86 & ME2PL & $\mathrm{Norm}_{2}$ & - & 8 \\
\hline NGC $5813^{*}(\mathrm{X})$ & Non-AGN & 41.33 & 40.30 & 8.42 & -4.72 & MEPL & - & - & 4 \\
\hline$(\mathrm{C})$ & & 39.68 & 39.07 & 8.42 & -5.95 & MEPL & - & - & 6 \\
\hline NGC $5982(\mathrm{X})$ & & 40.70 & 40.49 & 8.44 & -4.71 & MEPL & $\mathrm{Norm}_{2}$ & - & 1 \\
\hline $\mathrm{NGC} 4552^{A}(\mathrm{X}, \mathrm{C})$ & $\mathrm{AGN}$ & 39.49 & 39.45 & 8.84 & -6.00 & MEPL & Norm $_{1}$ & Norm $_{2}$ & 2 \\
\hline $\mathrm{NGC} 5846^{A}(\mathrm{X})$ & Non-AGN & 40.29 & 39.33 & 8.49 & -5.74 & MEPL & - & - & 7 \\
\hline$(\mathrm{C})$ & & & & & & MEPL & - & - & 0.6 \\
\hline \multicolumn{10}{|c|}{ Type 1} \\
\hline NGC $315(\mathrm{C})$ & $\mathrm{AGN}$ & 41.38 & 41.58 & 8.65 & -3.67 & MEPL & - & - & 3 \\
\hline NGC $1052(\mathrm{X})$ & $\mathrm{AGN}$ & 41.04 & 41.52 & 8.07 & -3.14 & ME2PL & $\mathrm{Norm}_{2}$ & $N_{H 2}$ & 8 \\
\hline NGC $2681^{*}(\mathrm{C})$ & $\mathrm{AGN}$ & 39.02 & 40.35 & 7.07 & -3.31 & MEPL & - & - & 0.4 \\
\hline NGC $3226(\mathrm{X}, \mathrm{C})$ & $\mathrm{AGN}$ & 40.78 & 41.01 & 8.22 & -3.80 & $2 \mathrm{PL}$ & $N_{H 2}$ & $\mathrm{Norm}_{2}$ & 1 \\
\hline NGC $3718(\mathrm{C}+\mathrm{X})$ & $\mathrm{AGN}$ & 40.76 & 40.99 & 7.85 & -3.60 & $2 \mathrm{PL}$ & $\mathrm{Norm}_{2}$ & - & 1 \\
\hline NGC $4278(\mathrm{C})$ & $\mathrm{AGN}$ & 39.80 & 39.75 & 8.46 & -5.30 & MEPL & $\mathrm{Norm}_{2}$ & - & 1 \\
\hline NGC $4636^{*}(\mathrm{X})$ & Non-AGN & 40.86 & 40.28 & 8.16 & -5.00 & MEPL & $\Gamma$ & - & 0.5 \\
\hline
\end{tabular}

A Objects from Hernández-García et al. (2013)

Table 1: Results from the spectral fitting in LINERs. Name (the * represent Compton-thick objects), and instrument (Col. 1), type (Col. 2), logarithm of the soft and hard X-ray luminosities in $\mathrm{erg} \mathrm{s}^{-1}$ (Col. 3 and 4), black-hole mass (Col. 5), Eddington ratio (Col. 6), best fit for the SMF0 (Col. 7), parameter varying in SMF1 (Col. 8), parameter varying in SMF2 (Col. 9), and the sampling timescale for variations (Col. 10). 


\section{References}

D'onofrio, M., Marziani, P., \& Sulentic, J. W. 2012, Fifty Years of Quasars: Current Impressions and Future Perspectives. Astrophysics and Space Science Library, Vol. 386. Springer-Verlag Berlin Heidelberg

Dudik, R. P., Satyapal, S., Gliozzi, M., \& Sambruna, R. M. 2005, ApJ, 620, 113

Evans, D. A., Hardcastle, M. J., Croston, J. H., Worral, D. M., \& Birkinshaw, M. 2005, MNRAS, 359,363

Eracleous, M., Hwang, J. A., \& Flohic, H. M. L. G. 2010, ApJs, 187, 135

González-Martín, O., Masegosa, J., Márquez, I., Guainazzi, M., \& Jiménez-Bailón, E. 2009, $A \mathscr{E} A, 506,1170$

González-Martín, O., Papadakis, I., Braito, V., Masegosa, J., Márquez, I., Mateos, S., AcostaPulido, J. A., Martínez, M. A., Ebrero, J., Esquej,P., O’Brien, P., Tueller, J., Warwick, R. S., \& Watson, M. G. 2011, $A \mathscr{E} A$, 527, A142

González-Martín, O. \& Vaughan, S. 2012, A\& $A$, 544, A80

Hernández-García, L., González-Martín, O., Márquez, I., \& Masegosa, J. 2013, A 6 A, 556, A47

Ho, L. C., Filippenko, A. V., Sargent,W. L. W., \& Peng, C. Y. 1997, ApJS, 112, 391

Ho, L. C. 2008, ARA\&A, 46, 475

Maoz, D., Nagar, N. M., Falcke, H., \& Wilson, A. S. 2005, ApJ, 625, 699

Masegosa, J., Márquez, I., Ramirez, A., \& González-Martín, O., 2011, A\&̧A, 527, A23

Pian, E., Romano, P., Maoz, D., Cucchiara, A., Pagani, C., \& Parola, V. L. 2010, MNRAS, 401, 677

Risaliti, G., Elvis, M., Bianchi, S., \& Matt, G. 2010, MNRAS, 406, L20

Satyapal, S., Sambruna, R. M., \& Dudik, R. P. 2004, A\&GA, 414, 825

Satyapal, S., Dudik, R. P., O'Halloran, B., \& Gliozzi, M. 2005, ApJ, 633, 86

Younes, G., Porquet, D., Sabra, B., \& Reeves, J. N., 2011 A\&A, 530, A149 